\title{
Efeito da ordem dos exercícios no número de repetições e na percepção subjetiva de esforço em homens treinados em força
}

CDD. 20.ed. 796.071

796.073

\author{
Saulo GIL* \\ Hamilton ROSCHEL** \\ Mauro BATISTA* \\ Carlos UGRINOWITSCH* \\ Valmor TRICOLI* \\ Renato BARROSO***
}

*Universidade de Ribeirão Preto.

**Escola de Educação Física e Esporte,

Universidade de São Paulo.

\section{Resumo}

A ordem dos exercícios refere-se à sequência de execução durante uma sessão de treinamento. Evidências demonstram que essa ordem pode afetar o número de repetições realizadas nos exercícios. A percepção subjetiva de esforço (PSE), assim como o número de repetições realizadas, depende da sobrecarga utilizada. Assim, alterações no número de repetições podem afetar a PSE. 0 volume total de trabalho (VTT) influencia nas adaptações crônicas ao treinamento e também pode ser afetado pela ordem dos exercícios. 0 objetivo foi verificar o efeito da ordem dos exercícios para membros inferiores no número de repetições realizadas, na PSE e no VTT. Doze homens treinados $(19,3 \pm 2,1$ anos, 71,1 $\pm 9,8 \mathrm{~kg}, 172,4 \pm 6,1 \mathrm{~cm}, 23,3$ $\pm 11,5$ meses/treino) realizaram duas sessões com os exercícios "leg-press" (L), mesa flexora (F) e cadeira extensora (E) em diferentes ordens (LFE ou EFL). Foram utilizados testes t de "Student" pareados com ajuste de Bonferroni para comparações múltiplas. 0 número de repetições em $L$ e $E$ diminuiu quando realizados no final da sessão. As repetições realizadas em F diminuíram na LFE. A PSE de E foi maior quando realizada no final da sessão, porém de $L$ e de $F$ não foram afetadas pelas diferentes ordens. 0 volume de trabalho total de LFE foi maior. Em conclusão, a ordem dos exercícios envolvendo membros inferiores afeta o número de repetições e a PSE de um exercício além do VTT, ressaltando a importância da ordem dos exercícios como uma importante variável na prescrição do treinamento.

UnIteRmos: Treino de força; Volume total; Hipertrofia.

\section{Introdução}

Quando um estímulo como o treinamento de força é aplicado ao músculo esquelético, as principais adaptações são o aumento da força e do tamanho (hipertrofia) do músculo. Essas adaptaçóes podem ser moduladas através da manipulação das variáveis do treinamento, tais como, número de séries e repetiçōes, intensidade de carga, volume de treino, escolha e ordem dos exercícios (FLecK \& KRAEMER, 2004; RAtamess, Alvar, Evetoch, Housh, Kibler, Kraemer \& Triplett, 2009). A ordem dos exercícios refere-se à sequência em que eles são realizados em uma sessão de treinamento (Simão, FarinatTi, Polito, Viveiros \& FLECK, 2007), a qual parece influenciar diretamente o número de repetições e consequentemente o volume total de trabalho (VTT) (GentiL, Oliveira, De Araujo Rocha Junior, Do Carmo \& Bottaro, 2007; Monteiro, Simão \& Farinatti, 2005; SimÃo, Farinatti, Polito, Maior \& Fleck, 2005; Simāo et al., 2007; Spreuwenberg, Kraemer, Spiering, Volek, Hatfield, Silvestre, Vingren, Fragala, Hakkinen, Newton, Maresh \& Fleck, 2006).

Sabe-se que o número de repetições realizadas com uma mesma carga relativa está relacionado com o tamanho do grupo muscular envolvido no exercício (Shimano, Kraemer, Spiering, Volek, Hateield, Silvestre, Vingren, Fragala, Maresh, Fleck, Newton, Spreuwenberg \& Hakkinen, 2006). Assim, tem sido sugerido que a ordem de 
realização dos exercícios poderia afetar de maneira a distinta o número de repetições realizadas em grupos musculares de tamanhos diferentes (SFORZO \& Touey, 1996). Entretanto, após submeterem seus participantes a duas sessóes de treino em que exercícios para grandes e pequenos grupos musculares dos membros superiores e inferiores eram realizados em ordens diferentes, SFORZO e Touey (1996) observaram que o número de repetições no último exercício realizado nas sessōes, era significantemente reduzido. Dessa maneira, a hipótese de que a ordem afetaria o número de repetições de maneira dependente do tamanho do grupo muscular foi desconsiderada. Diferentemente de Sforzo e Touey ( (1996), Simâo et al. (2007) propuseram que o número de articulações envolvidas no exercício, e não o tamanho do grupo muscular influenciaria o número de repetiçôes realizadas. Baseado nesse pressuposto, Simāo et al. (2007) utilizaram exercícios mono e multiarticulares envolvendo membros superiores e inferiores em duas ordens distintas e observaram que independente do número de articulaçôes envolvidas, quando o exercício era realizado no final da sessão o número de repetiçōes era significantemente reduzido. Portanto, a hipótese de que o número de articulações seria um fator importante para o número de repetiçōes também foi rejeitada.

Os resultados desses estudos aplicam-se a programas que envolvam exercícios para membros superiores e membros inferiores, na mesma sessão de treinamento. Contudo, é recomendado que os indivíduos com maior tempo de treinamento realizem exercícios para segmentos corporais distintos, em dias separados (ex. "split routine") (Kraemer, Adams, Cafarelli, Dudley, Dooly, Feigenbaum, Fleck, Franklin, Fry, Hoffman, Newton, Potteiger, Stone, Ratamess \& TriplettMcBride, 2002; Ratamess et al., 2009). Nessa divisão, exercícios para membros superiores e inferiores seriam realizados em dias diferentes (Kraemer et al., 2002; Ratamess et al., 2009). Dessa maneira, é importante entender como a ordem dos exercícios afeta o número de repetições quando apenas exercícios para membros superiores ou inferiores são realizados.

Outros estudos foram realizados investigando o efeito da ordem dos exercícios no número de repetições realizadas envolvendo apenas membros superiores (Gentil et al., 2007; Monteiro, SimĀo \& FARINATTI, 2005; SimÃo et al., 2005). Os resultados observados com membros superiores corroboram com os encontrados em sessões que envolviam membros superiores e inferiores (SForzo \& TOUEY, 1996; SIMÃo et al., 2007), ou seja, o número de repetiçōes nos exercícios realizados no final da sessão de treinamento era reduzido.

Considerando que o número de repetições realizadas com uma mesma carga relativa é menor em exercícios para membros superiores do que em exercícios para membros inferiores (SHIMANO et al., 2006), é possível que a ordem dos exercícios afete o comportamento do número de repetiçōes distintamente em membros inferiores e superiores. No entanto, até o momento é desconhecido o efeito da ordem quando apenas exercícios para membros inferiores são realizados.

Além disso, sugere-se que a manipulação da ordem dos exercícios afete o VTT ao influenciar o número de repetições realizadas (MonteIro, Simão $\&$ FarinatTI, 2005; SHIMANO et al., 2006; SimĀo et al., 2005). O VTT é obtido pela multiplicação do número de séries e repetições pela carga utilizada em cada exercício (RATAMEss et al., 2009). Diferentes VTT vêm sendo associados a respostas agudas distintas (Gotshalk, Loebel, Nindl, Putukian, Sebastianelli, Newton, Hakkinen \& Kraemer, 1997; Kraemer, Marchitelli, Gordon, Harman, Dziados, Mello, Frykman, McCurry \& Fleck, 1990; Ratamess, Falvo, Mangine, Hoffman, FAigenbaUm \& KanG, 2007), que podem influenciar as adaptaçóes crônicas ao treinamento (hipertrofia/ ganho de força) (Tesch, Komi \& HakKinen, 1987). No entanto, o efeito da manipulação da ordem dos exercícios sobre o VTT da sessão ainda não foi investigado.

Além disso, alguns estudos mostraram que a percepção subjetiva de esforço (PSE), ao final de uma sessão de treinamento, não variava em função da ordem de realização dos exercícios (Monteiro, SiMÃo \& Farinatti, 2005; SimÃo et al., 2005, 2007). Contudo, considerando que o número de repetições realizadas em um determinado exercício (SHIMANO et al., 2006), assim como a PSE (SWEet, Foster, MCGUigan \& BRICE, 2004), são dependentes da sobrecarga utilizada, é esperado que qualquer alteração no número de repetiçôes para uma determinada carga fixa seja refletida também na PSE. Deste modo, o objetivo do presente estudo foi verificar o efeito da ordem de realização dos exercícios de membros inferiores sobre o número de repetições, sobre a PSE de cada exercício e da sessão de treinamento, bem como avaliar a influência da ordem sobre o VTT realizado na sessão. 


\section{Materiais e método}

\section{Amostra}

Doze voluntários do sexo masculino (19,3 \pm 2,1 anos, 71,1 $\pm 9,8 \mathrm{~kg}, 172,4 \pm 6,1 \mathrm{~cm})$ com no mínimo 12 meses de experiência $(3 \mathrm{x} /$ semana) em treinamento de força para membros inferiores (23,3 $\pm 11,5$ meses $)$, sem histórico de lesão articular no quadril, joelho e tornozelo, participaram do estudo. Todos os sujeitos foram informados sobre os objetivos e riscos da pesquisa e assinaram um consentimento livre e esclarecido sobre os riscos e benefícios associados a sua participação no estudo. Este estudo foi aprovado pelo Comitê de Ética em Pesquisa da Escola de Educação Física e Esporte da Universidade de São Paulo (protocolo no 2009/49).

\section{Procedimentos experimentais}

Os indivíduos foram testados para verificar o número de repetiçôes máximas utilizando uma carga de $80 \%$ do máximo (1 RM) em duas ordens distintas. Uma das ordens utilizadas foi "leg press", mesa flexora e cadeira extensora (LFE) e a outra ordem foi cadeira extensora, mesa flexora e "leg press" (EFL). Os participantes realizaram uma sessão de familiarização e cinco sessões experimentais. Uma semana antes do início dos testes, os participantes foram familiarizados com os procedimentos dos testes nos três exercícios. A ordem dos exercícios na sessão de familiarização foi aleatorizada entre os participantes. Durante as três primeiras sessões experimentais, os participantes realizaram testes de força dinâmica máxima (1 RM) em cada um dos exercícios. Essas sessões foram separadas por pelo menos 48 h. Nas outras sessões experimentais, foi avaliado o número máximo de repetiçôes realizadas em três séries em cada exercício, o número total de repetiçôes realizadas nos três exercícios e o VTT nas diferentes ordens. O VTT foi calculado somando o volume de cada exercício. Assim, o VTT = (volume do "leg-press" + volume da cadeira extensora + volume da mesa flexora). O volume de trabalho de cada exercício foi calculado multiplicando o número de séries (três) pelo número de repetiçôes (avaliado em cada uma das ordens) e pela carga utilizada (em kg). Após a realização de cada exercício foi apresentada a escala de CR-Borg-10 (DAY, McGuigan, BRICE $\&$ Foster, 2004) ao participante e pedido para que ele indicasse o número que melhor representava o esforço realizado.

\section{Teste de força dinâmica máxima (1 RM)}

Os testes de 1 RM seguiram os procedimentos propostos pela Sociedade Americana de Fisiologistas do Exercício (BROWN \& WEIR, 2001). Os participantes realizaram um aquecimento geral em esteira rolante a $9 \mathrm{~km} / \mathrm{h}$ durante cinco minutos, seguido de cinco minutos de exercícios de flexibilidade para os membros inferiores e por um aquecimento especifico. O aquecimento específico foi composto de uma série de cinco repetiçôes com aproximadamente 50\% 1 RM (estimado na sessão de familiarização) e uma série de três repetições com aproximadamente $80 \% 1 \mathrm{RM}$, com dois minutos de intervalo entre as séries. Três minutos após o término do aquecimento, foi iniciado o teste de 1 RM. A força dinâmica máxima foi determinada no máximo em cinco tentativas com três minutos de intervalo entre elas.

O exercício "leg press" iniciou-se com a extensão total dos joelhos. A partir dessa posição, os participantes deveriam flexionar os joelhos e o quadril, até atingirem a posição de $90^{\circ}$ de flexão determinada nas sessões de familiarização. Para que os sujeitos identificassem a amplitude correta do movimento durante o teste, uma marcação foi colocada ao longo do trilho onde a plataforma de apoio dos pés deslizava. Ao atingirem essa marcação, os participantes deveriam estender os joelhos até a posição inicial, finalizando o ciclo do exercício.

$\mathrm{Na}$ cadeira extensora, o ciclo do movimento foi iniciado com os joelhos em um ângulo de $180^{\circ}$. Ao sinal do avaliador o sujeito realizava a fase excêntrica flexionando os joelhos até alcançar o ângulo de $90^{\circ}$ e depois os estendia até alcançar novamente a posição inicial.

$\mathrm{Na}$ mesa flexora, o ciclo de movimento iniciouse com os joelhos em um ângulo de $90^{\circ}$ e ao sinal do avaliador o sujeito realizava a fase excêntrica do movimento estendendo os joelhos até alcançar o ângulo de $180^{\circ}$ e depois os flexionava novamente até a posição inicial.

Os participantes foram encorajados verbalmente durante a realização das tentativas. Foram consideradas válidas apenas as repetições que cumpriram o ciclo completo de movimento.

\section{Sessões experimentais}

Todos os participantes realizaram as duas ordens propostas, LFE e EFL, em dias diferentes separados por pelo menos $48 \mathrm{~h}$. A ordem de realização das 
sequências foi randomizada entre os participantes. Todos eles foram instruídos a não realizarem nenhum exercício ou atividade física extenuante $24 \mathrm{~h}$ antes das sessōes experimentais.

Cada sessão experimental foi iniciada com um aquecimento geral em esteira rolante a $9 \mathrm{~km} / \mathrm{h}$. Algumas evidências mostram que exercícios de flexibilidade estática de alta intensidade realizados antes de uma sessão de treinamento podem reduzir a capacidade de gerar força máxima (BACURAU, Monteiro, Ugrinowitsch, Tricoli, Cabral \& AоKI, 2009). Desta forma, os participantes foram instruídos a não realizar exercícios de flexibilidade com essas características antes dos testes de $1 \mathrm{RM}$ e das sessões experimentais. Três minutos após o aquecimento geral foi ajustada a carga em $80 \% 1$ RM e cada participante realizou em cada exercício três séries até a falha concêntrica. Foi respeitado um intervalo de dois minutos entre cada série e entre

\section{Resultados}

Os valores de 1 RM para os três exercícios, assim como seus respectivos valores utilizados para a realização das sessões de treinamento, são apresentados na TABELA 1. Não houve diferença significante no número total de repetiçôes realizadas (soma das repetições nas três séries dos três exercícios) entre as sequências ( $\mathrm{LFE}=57,5 \pm 7,9$ e $\mathrm{EFL}=61,3$ $\pm 7,8, \mathrm{t}=1,65, \mathrm{p}=0,13$, FIGURA 1 à direita). Contudo, quando considerados os exercícios isoladamente, houve diferenças significantes entre as sequências. $\mathrm{O}$ número de repetiçōes realizados no "leg press" foi significantemente maior na LFE do que na EFL ( $\mathrm{LFE}=31,8 \pm 4,8$ e EFL $=21,7 \pm 3,9$, $\mathrm{t}=4,44, \mathrm{p}<0,001)$; na cadeira extensora o número de repetições realizadas foi maior na EFL comparado com a LFE $(\mathrm{LFE}=12,9 \pm 4,2$ e EFL $=24,9 \pm 4,4, \mathrm{t}$ $=8,24, \mathrm{p}<0,001)$ e na mesa flexora foram realizadas exercícios. Foram contadas apenas as repetições que completaram um ciclo de movimento. Os participantes foram encorajados verbalmente durante a realização dos exercícios.

\section{Análise estatística}

Os resultados são apresentados através de estatística descritiva (média \pm desvio padrão). Os dados foram testados para normalidade através do teste de Komolgorov-Smirnov. O número de repetiçōes realizadas, o VTT (série $\mathrm{x}$ repetições $\mathrm{x}$ carga) e a percepção subjetiva de esforço em cada exercício nas duas sequências foram testados pelo teste t de "Student" pareado. O nível de significância adotado foi de $\mathrm{p}<0,05$. Devido à realização de múltiplas comparações, foi utilizada a correção de Bonferroni. Sendo assim, o nível de significância corrigido foi de $\mathrm{p}<0,00625$.

mais repetiçoes durante a $E F L(L F E=12,6 \pm 2,7$ e $\mathrm{EFL}=14,6 \pm 3,2, \mathrm{t}=3,94, \mathrm{p}=0,002)$ (FIGURA 1).

Considerando que a PSE do ultimo exercício pode refletir a PSE da sequência pode-se dizer que não houve diferença estatística entre as sequências $(\mathrm{LFE}=9,4 \pm 0,1$ e EFL $=9,2 \pm 0,1, \mathrm{t}=1,77, \mathrm{p}=$ $0,10)$. A PSE na cadeira extensora foi maior durante a LFE $(\mathrm{LFE}=9,8 \pm 0,2$ e EFL $=8,8 \pm 3,9, \mathrm{t}=4,69, \mathrm{p}$ $<0,001)$, não houve diferenças significantes no "legpress" $(\mathrm{LFE}=8,7 \pm 0,2$ e EFL $=9,5 \pm 0,2, \mathrm{t}=2,46$, $\mathrm{p}=0,03)$, nem na mesa flexora $(\mathrm{LFE}=9,5 \pm 0,2 \mathrm{e}$ $\mathrm{EFL}=9,4 \pm 0,2, \mathrm{t}=1,48, \mathrm{p}=0,16)($ FIGURA 2).

A análise do VTT demonstrou que apesar de não haver diferenças no número total de repetições, o volume total da LFE $(7.341,7 \pm 1.599,5 \mathrm{~kg})$ foi significantemente maior do que o da EFL (6.384,5 $\pm 1.152,8)($ FIGURA 3, $\mathrm{t}=3,96, \mathrm{p}<0,001)$.

TABELA 1- Valores de 1 RM (média \pm dp) e da carga utilizada nos três exercícios utilizados.

\begin{tabular}{cccc}
\hline & “Leg-press” & Cadeira Extensora & Mesa flexora \\
\hline 1 RM $(\mathrm{kg})$ & $248,3 \pm 38,1$ & $59,8 \pm 10,6$ & $27,4 \pm 4,1$ \\
$80 \% 1 \mathrm{RM}(\mathrm{kg})$ & $203,0 \pm 35,2$ & $48,0 \pm 8,4$ & $21,9 \pm 3,2$ \\
\hline
\end{tabular}




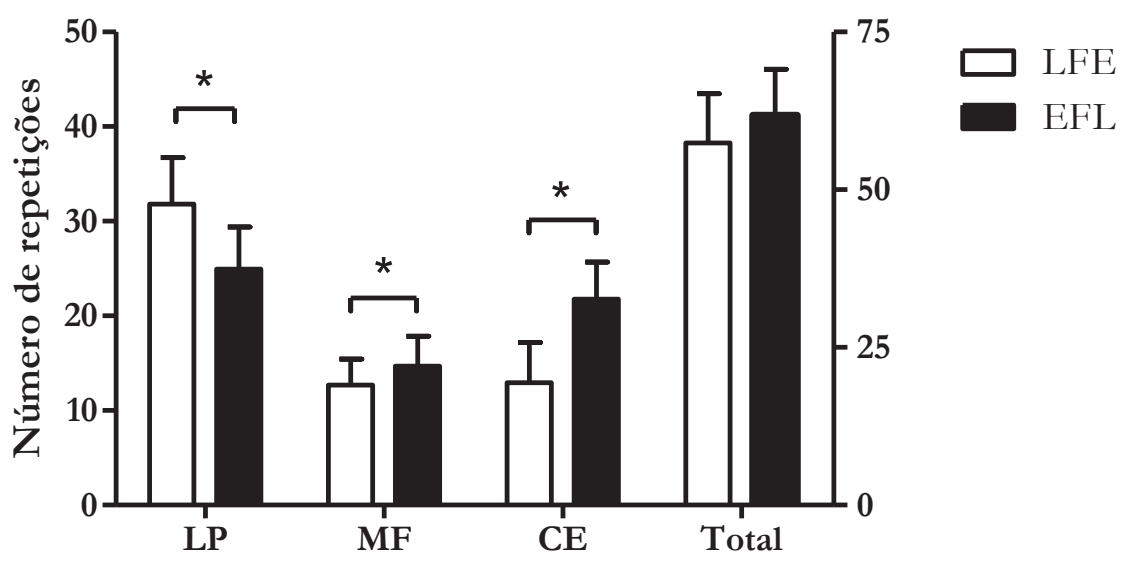

${ }^{\star} p<0,00625$.

FIGURA 1 - Número derepetições (média _desvio padrão) realizadas nos exercícios “leg-press” (LP), mesa flexora (MF) e cadeira extensora (CE) e o número total de repetições na sessão nas sequências LFE e EFL .

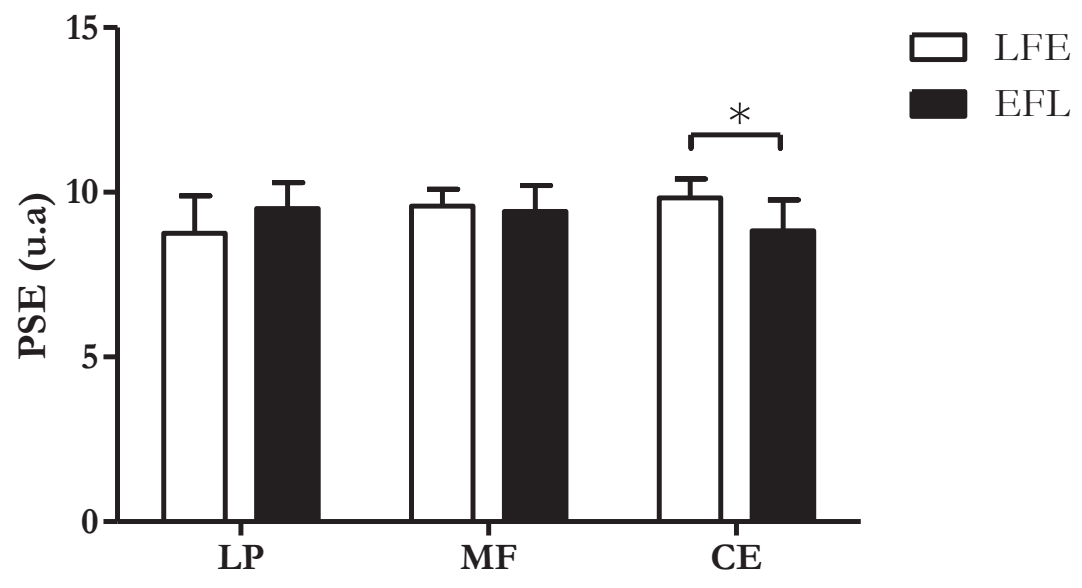

${ }^{*} p<0,00625$.

FIGURA 2 - Percepção subjetiva de esforço (u.a.), (média \pm desvio padrão) após os exercícios "leg-press” (LP), mesa flexora (MF) e cadeira extensora (CE) e a percepção de esforço da sessão em duas sequências diferentes.

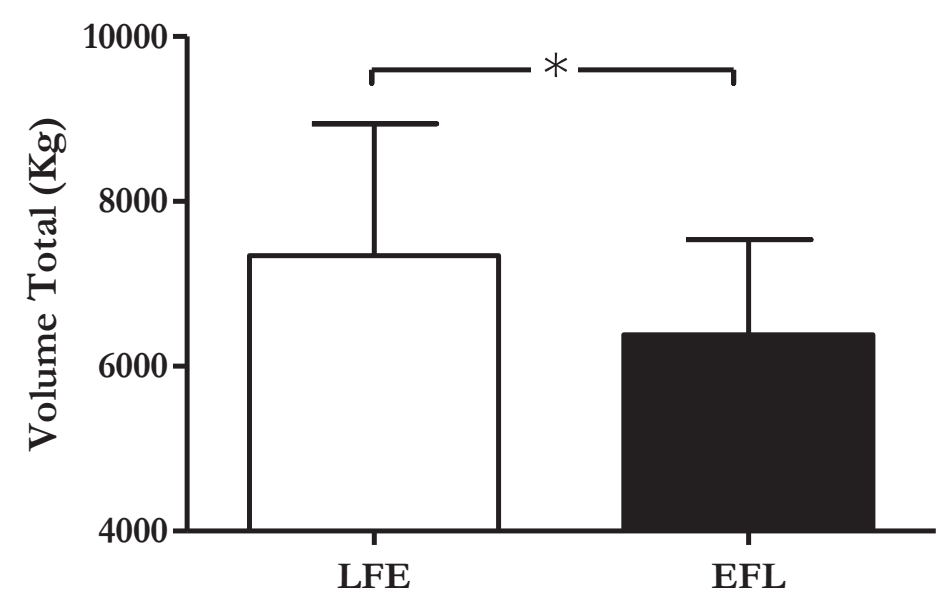

${ }^{*} \mathrm{p}<0,001$.

FIGURA 3 - Volume total de trabalho (média \pm desvio padrão) nas duas sequências de exercícios.

Rev. bras. Educ. Fís. Esporte, São Paulo, v.25, n.1, p.127-35, jan./mar. 2011 • 131 


\section{Discussão}

O objetivo desse estudo foi investigar se a ordem de realização dos exercícios de força em uma sessão de treino envolvendo apenas membros inferiores afetaria o número de repetições e a percepção subjetiva de esforço (PSE). Nossos resultados demonstram que: 1) o número de repetições em um exercício é menor quando este é realizado no final da sessão de treinamento, mas a realização dos exercícios em diferentes ordens não afeta o número total de repetições da sessão; 2) quando exercícios multiarticulares são realizados no início da sessão, a PSE dos exercícios subsequentes para o mesmo grupo muscular é significantemente maior; no entanto, quando exercícios uniarticulares são realizados primeiramente, a PSE dos exercícios subsequentes não sofre alterações; e 3) a ordem dos exercícios afeta o VTT da sessão de treinamento.

Algumas hipóteses foram sugeridas na tentativa de explicar como a ordem dos exercícios poderia influenciar o número de repetições realizadas. Sforzo e Touey (1996) sugeriram que a ordem dos exercícios afetaria o número de repetições realizadas de maneira dependente do tamanho do grupo muscular. SIMÃo et al. (2007) propuseram que o número de articulaçōes envolvidas e não o tamanho do grupo muscular seria o fator determinante em como a ordem afetaria o número de repetiçóes. Entretanto, nenhum destes estudos confirmou as hipóteses propostas; pois, em sessóes que envolviam exercícios para membros superiores e inferiores, independente do tamanho do músculo ou do número de articulações envolvidas, o exercício realizado ao final da sessão tinha o número de repetiçóes reduzido. No presente estudo, apesar de termos utilizado os mesmos exercícios para membros inferiores dos estudos supracitados (SForzo \& ToueY, 1996; SIMÃo et al., 2007), eles foram aplicados em uma sequência diferente. Propusemos a realização de um exercício em que o quadríceps não fosse o agonista (mesa flexora) entre os dois exercícios para este grupo muscular ("leg-press" e cadeira extensora), de forma a induzir um maior tempo de descanso e possivelmente influenciar positivamente a manutenção do número de repetiçôes, e consequentemente do volume de treinamento. Embora a estratégia adotada tenha sido efetiva em aumentar o tempo de descanso, este aumento não foi suficiente para garantir a manutenção do número de repetições no exercício realizado subsequentemente. De fato, nossos resultados demonstram que quando um exercício é realizado no final da sessão, o número de repetições realizadas é menor, independente do número de articulações ou grupos musculares envolvidos, corroborando com resultados prévios na literatura (SForzo \& Touey, 1996; SimÃo et al., 2007).

Além disso, foi também possível observar que o número de repetições realizado na mesa flexora (colocada no meio da sessão) foi afetado pela ordem. Quando realizado após o "leg press", o número de repetiçôes completadas foi menor do que quando o mesmo exercício foi precedido pela cadeira extensora. Essas observações são contrárias às de SIMÃo et al. (2005) que investigaram a influência da ordem dos exercícios apenas para membros superiores (supino, puxador frente, desenvolvimento, rosca direta e extensão de cotovelo). Os autores não observaram diferença no número de repetiçōes do exercício que era realizado no meio da sessão (desenvolvimento), e atribuiram seus achados ao tempo de descanso aumentado, uma vez que exercícios consecutivos não utilizavam os mesmos grupos musculares. Entretanto, é possível que no presente estudo, embora a mesa flexora tenha os músculos posteriores como agonistas, algum nível de fadiga tenha sido induzido nesse grupo muscular uma vez que o "leg-press" promove ativação dos músculos posteriores da coxa durante a extensão do quadril (Da Silva, Brentano, Cadore, De Almeida \& Kruel, 2008). Outra possibilidade para explicação dos achados está relacionada com a quantidade aumentada de grupos musculares envolvidos. Isto pode ter gerado maior grau de fadiga e provocado a diminuição no número de repetições nos exercícios subsequentes (Monteiro, SimÃo \& FarinatTI, 2005). Contudo, neste cenário seria esperada, então, diferença na PSE após o primeiro exercício de cada sequência, que de fato não ocorreu.

Recentemente, alguns estudos têm mostrado que a PSE pode refletir a intensidade do exercício durante sessões de treinamento de força (DAY et al., 2004; Lagally, Robertson, Gallagher, Gearhart \& Goss, 2002; LAgally, RoBERTSON, Gallagher, Goss, Jakicic, Lephart, McCaW \& Goodpaster, 2002). Três estudos investigaram a PSE após a realização de diferentes sequências de exercícios (MONTEIRO, SiMĀo $\&$ FARINATTI, 2005; SimÃo et al., 2005, 2007) e não observaram diferenças entre as sequências. Contudo, diferente dos anteriores, o presente estudo procurou verificar se a ordem afetaria além do número de repetições, também a PSE do exercício. Sabe-se que a PSE, assim como o número de repetiçôes (SHIMANO et al., 2006), é relacionada com a intensidade relativa do exercício (SwEET et al., 2004). A PSE na cadeira 
extensora foi maior quando o exercício foi realizado no final da sequência. Na mesa flexora, exercício que sempre esteve no meio da sessão, não foi observado diferença significante na PSE, independentemente da sequência realizada. Portanto, se a PSE reflete a intensidade do exercício, então nossos resultados sugerem que quando o exercício é realizado no final da sequência a sua intensidade parece ser maior, possivelmente por uma diminuição na capacidade de produção de força máxima.

Nossos resultados apontam ainda para uma diferença no VTT realizado na sessão de treinamento em consequência da ordem. A execução de exercícios que envolviam um número maior de articulações no início da sessão influenciou positivamente o VTT da sessão. Este achado se torna particularmente importante à medida que a literatura aponta o efeito positivo de maiores VTT sobre o ganho de força (Hass, Garzarella, De Hoyos \& Pollock, 2000; Paulsen, Myklestad \& RaAstad, 2003; Kelly, Brown, Coburn, Zinder, Gardner \& Nguyen, 2007) e hipertrofia (Hansen, Kvorning, KJaer \& SJogaArd, 2001; Ronnestad, Egeland, Kvamme, Refsnes, KADI \& RAASTAD, 2007) de membros inferiores. Similarmente, Burd, West, Staples, Atherton, Baker, Moore, Holwerda, Parise, Rennie, BaKer e Phillips (2010) observaram que a resposta aguda de síntese protéica, especialmente miofibrilar, está relacionada ao volume de treinamento. Além disso, Gotshalk et al. (1997), Hansen et al. (2001) e West, BurD, TANG, Moore, Staples, Holwerda, BaKer e Phillips (2010) mostraram que um maior VTT induz maior liberação de hormônios anabólicos como testosterona e GH. Assim, é possível que treinamento com maior volume seja mais eficiente do que com menor volume para induzir ganhos de força e/ou hipertrofia.

De fato, foi observado maior hipertrofia e ganho de força dos flexores do cotovelo em um grupo que realizava exercícios para os membros inferiores previamente ao treinamento dos flexores de cotovelo (HANSEN et al., 2001). Os autores atribuíram esses resultados à maior liberação de hormônios anabólicos (testosterona e GH) induzida pelo maior VTT realizado pelo grupo que exercitou os membros inferiores além dos membros superiores. No entanto, apesar de terem observado diferentes respostas hormonais, WEST et al. (2010) não observaram diferenças no ganho de força e hipertrofia quando compararam dois modelos de treino similares aos utilizados por HANSEN et al. (2001). Contudo, West et al. (2010) realizaram exercícios de membros inferiores após o exercício para membros superiores, evidenciando, novamente, o papel da ordem de realização dos exercícios.

Desta forma, analisando conjuntamente os dados citados acima junto com os nossos, é possível sugerir que exercícios multiarticulares devam ser realizados no início da sessão, uma vez que permitirá o volume total da sessão ser maior e possivelmente induzir maiores ganhos de força e hipertrofia. Essa sugestão corrobora com as recomendaçôes do American College of Sports Medicine (RATAMESs et al., 2009).

Em conclusão, a manipulação da ordem em uma sessão de treinamento de força utilizando apenas membros inferiores afeta o número de repetições realizadas em determinado exercício. Quando um exercício é realizado no final da sessão, o número de repetiçôes é reduzido. No entanto, o número de repetiçôes de um exercício localizado no meio da sessão parece ser negativamente influenciado quando precedido de um exercício multiarticular que envolve grandes grupos musculares. Quando analisada a PSE de exercícios para o mesmo grupo muscular agonista nas duas sequências, esta é aumentada quando exercícios multiarticulares são realizados no início da sessão. Já o volume total realizado na sessão é afetado pela ordem dos exercícios, e a realização de exercícios multiarticulares no início da sessão parece influenciar positivamente o volume total.

\section{Abstract}

\section{The effect of the exercises order on number of repetitions and rate of perceived effort in resistance trained men}

The order of exercises refers to the sequence during a training bout. Some evidence shows that the number of repetitions may be affected by the order of exercises. Both the number of repetitions and the rate of perceived effort (PSE) are influenced by exercise load. Thus, changes in the number of repetitions may affect PSE. Additionally, total work volume influences long term training adaptations and can be affected by the order of exercises. The aim of this study was to evaluate the effect of order of lower limb exercises on the number of repetitions, RPE and total work volume. Twelve resistance trained male (19.3 \pm 2.1 years, $71.1 \pm 9.8 \mathrm{~kg}, 172.4$ 
$\pm 6.1 \mathrm{~cm}, 23.3 \pm 11.5$ months of training) performed leg-press (L), leg extension (E) and leg curl (F) exercises, in different orders (LFE or EFL). Number of repetitions, PSE and total work volume were compared by paired t-test with Bonferroni adjustment. Number of repetitions on both $L$ and $E$ decreased when they were performed last in the bout. The number of repetitions on F also decreased, when F was preceded by L. PSE for $E$ was greater when performed at the end of the bout. However, PSE after $L$ and $F$ were not affected by the order. Total work volume was greater in LFE. In conclusion, the order of lower limb exercises affects the number of repetitions, PSE and total work performed, supporting the importance of the order of exercises as an important variable in training prescription.

UNITERMS: Resistance training; Total volume; Hypertrophy.

\section{Referências}

BACURAU, R.F.; MONTEIRO, G.A.; UGRINOWITSCH, C.; TRICOLI, V.; CABRAL, L.F.; AOKI, M.S. Acute effect of a ballistic and a static stretching exercise bout on flexibility and maximal strength. Journal of Strength and Conditioning Research, Champaign, v.23, n.1, p.304-8, 2009.

BROWN, L.; WEIR, J. ASEP procedures recommendation I: accurate assessment of muscular strength and power. Journal of Exercise Physiology, Duluth, v.4, n.3, p.1-21, 2001.

BURD, N.A.; WEST, D.W.; STAPLES, A.W.; ATHERTON, P. J.; BAKER, J.M.; MOORE, D.R.; HOLWERDA, A.M.; PARISE, G.; RENNIE, M.J.; BAKER, S.K.; PHILLIPS, S.M. Low-load high volume resistance exercise stimulates muscle protein synthesis more than high-load low volume resistance exercise in young men. PLoS One, San Francisco, v.5, n.8, p.e12033, 2010. DA SILVA, E.M.; BRENTANO, M.A.; CADORE, E.L.; DE ALMEIDA, A.P.; KRUEL, L.F. Analysis of muscle activation during different leg press exercises at submaximum effort levels. Journal of Strength and Conditioning Research, Champaign, v.22, n.4, p.1059-65, 2008.

DAY, M.L.; McGUIGAN, M.R.; BRICE, G.; FOSTER, C. Monitoring exercise intensity during resistance training using the session RPE scale. Journal of Strength and Conditioning Research, Champaign, v.18, n.2, p.353-8, 2004.

FLECK, S.; KRAEMER, W. Designing resistance training programs. Champaign: Human Kinetics, 2004.

GENTIL, P.; OLIVEIRA, E.; DE ARAUJO ROCHA JUNIOR, V.; DO CARMO, J.; BOTTARO, M. Effects of exercise order on upper-body muscle activation and exercise performance. Journal of Strength and Conditioning Research, Champaign, v.21, n.4, p.1082-6, 2007.

GOTSHALK, L.A.; LOEBEL, C.C.; NINDL, B.C.; PUTUKIAN, M.; SEBASTIANELLI, W.J.; NEWTON, R.U.; HAKKINEN, K.; KRAEMER, W.J. Hormonal responses of multiset versus single-set heavy-resistance exercise protocols. Canadian Journal of Applied Physiology, Champaign, v.22, n.3, p.244-55, 1997.

HANSEN, S.; KVORNING, T.; KJAER, M.; SJOGAARD, G. The effect of short-term strength training on human skeletal muscle: the importance physiologically elevated hormonal levels. Scandinavian Journal of Medicine \& Science in Sports, Copenhagen, v.11, n.6, p.347-54, 2001.

HASS, C.J.; GARZARELLA, L.; DE HOYOS, D.; POLLOCK, M.L. Single versus multiple sets in long-term recreational weightlifters. Medicine and Science in Sports and Exercise, Madison, v.32, n.1, p.235-42, 2000.

KELLY, S.B.; BROWN, L.E.; COBURN, J.W.; ZINDER, S.M.; GARDNER, L.M.; NGUYEN, D. The effect of single versus multiple sets on strength. Journal of Strength and Conditioning Research, Champaign, v.21, n.4, p.1003-6, 2007. KRAEMER, W.J.; ADAMS, K.; CAFARELLI, E.; DUDLEY, G.A.; DOOLY, C.; FEIGENBAUM, M.S.; FLECK, S.J.; FRANKLIN, B.; FRY, A.C.; HOFFMAN, J.R.; NEWTON, R.U.; POTTEIGER, J.; STONE, M.H.; RATAMESS, N.A.; TRIPLETT-McBRIDE, T. American College of Sports Medicine position stand. Progression models in resistance training for healthy adults. Medicine and Science in Sports and Exercise, Madison, v.34, n.2, p.364-80, 2002.

KRAEMER, W.J.; MARCHITELLI, L.; GORDON, S.E.; HARMAN, E.; DZIADOS, J.E.; MELLO, R.; FRYKMAN, P.; McCURRY, D.; FLECK, S.J. Hormonal and growth factor responses to heavy resistance exercise protocols. Journal of Applied Physiology, Bethesda, v.69, n.4, p.1442-50, 1990.

LAGALLY, K.M.; ROBERTSON, R.J.; GALLAGHER, K.I.; GEARHART, R.; GOSS, F.L. Ratings of perceived exertion during low- and high-intensity resistance exercise by young adults. Perceptual and Motor Skills, Missoula, v.94, n.3 Pt 1, p.723-31, 2002. 
LAGALLY, K.M.; ROBERTSON, R.J.; GALLAGHER, K.I.; GOSS, F.L.; JAKICIC, J.M.; LEPHART, S.M.; McCAW, S.T.; GOODPASTER, B. Perceived exertion, electromyography, and blood lactate during acute bouts of resistance exercise. Medicine and Science in Sports and Exercise, Madison, v.34, n.3, p.552-9; 2002.

MONTEIRO, W.; SIMÃO, R.; FARINATTI, P. Manipulação na ordem dos exercícios e sua influência sobre o número de repetições e percepção subjetiva de esforço em mulheres treinadas. Revista Brasileira de Medicina do Esporte, São Paulo, v.11, n.2, p.146-50, 2005.

PAULSEN, G.; MYKLESTAD, D.; RAASTAD, T. The influence of volume of exercise on early adaptations to strength training. Journal of Strength and Conditioning Research, Champaign, v.17, n.1, p.115-20, 2003.

RATAMESS, N.A.; ALVAR, B.A.; EVETOCH, T.K.; HOUSH, T.J.; KIBLER, W.B.; KRAEMER, W.J.; TRIPLETT, N.; T. American College of Sports Medicine position stand: progression models in resistance training for healthy adults. Medicine and Science in Sports and Exercise, Madison, v.41, n.3, p.687-708, 2009.

RATAMESS, N.A.; FALVO, M.J.; MANGINE, G.T.; HOFFMAN, J.R.; FAIGENBAUM, A.D.; KANG, J. The effect of rest interval length on metabolic responses to the bench press exercise. European Journal of Applied Physiology, Berlin, v.100, n.1, p.1-17, 2007.

RONNESTAD, B.R.; EGELAND, W.; KVAMME, N.H.; REFSNES, P.E.; KADI, F.; RAASTAD, T. Dissimilar effects of one- and three-set strength training on strength and muscle mass gains in upper and lower body in untrained subjects. Journal of Strength and Conditioning Research, Champaign, v.21, n.1, p.157-63, 2007.

SFORZO, G.; TOUEY, P. Manipulating exercise order affects muscular performance during a resistance exercise training session. Strength and Conditioning Research, Champaign, v.10, n.1, p.196??, 1996.

SHIMANO, T.; KRAEMER, W.J.; SPIERING, B.A.; VOLEK, J.S.; HATFIELD, D.L.; SILVESTRE, R.; VINGREN, J.L.; FRAGALA, M.S.; MARESH, C.M.; FLECK, S.J.; NEWTON, R.U.; SPREUWENBERG, L.P.; HAKKINEN, K. Relationship between the number of repetitions and selected percentages of one repetition maximum in free weight exercises in trained and untrained men. Journal of Strength and Conditioning Research, Champaign, v.20, n.4, p.819-23, 2006. SIMÃO, R.; FARINATTI, P.T.; POLITO, M.D.; MAIOR, A.S.; FLECK, S.J. Influence of exercise order on the number of repetitions performed and perceived exertion during resistance exercises. Journal of Strength and Conditioning Research, Champaign, v.19, n.1, p.152-6, 2005.

SIMÃO, R.; FARINATTI, P.T.; POLITO, M.D.; VIVEIROS, L.; FLECK, S.J. Influence of exercise order on the number of repetitions performed and perceived exertion during resistance exercise in women. Journal of Strength and Conditioning Research, Champaign, v.21, n.1, p.23-8, 2007.

SPREUWENBERG, L.P.; KRAEMER, W.J.; SPIERING, B.A.; VOLEK, J.S.; HATFIELD, D.L.; SILVESTRE, R.; VINGREN, J.L.; FRAGALA, M.S.; HAKKINEN, K.; NEWTON, R.U.; MARESH, C.M.; FLECK, S.J. Influence of exercise order in a resistance-training exercise session. Journal of Strength and Conditioning Research, Champaign, v.20, n.1, p.141-4, 2006.

SWEET, T.W.; FOSTER, C.; McGUIGAN, M.R.; BRICE, G. Quantitation of resistance training using the session rating of perceived exertion method. Journal of Strength and Conditioning Research, Champaign, v. 18, n.4, p.796-802, 2004. TESCH, P.A.; KOMI, P.V.; HAKKINEN, K. Enzymatic adaptations consequent to long-term strength training. International Journal of Sports Medicine, Sttugart, v.8, p.66-9, 1987. Supplement 1.

WEST, D.W.; BURD, N.A.; TANG, J.E.; MOORE, D.R.; STAPLES, A.W.; HOLWERDA, A.M.; BAKER, S.K.; PHILLIPS, S.M. Elevations in ostensibly anabolic hormones with resistance exercise enhance neither training-induced muscle hypertrophy nor strength of the elbow flexors. Journal of Applied Physiology, Bethesda, v.108, n.1, p.60-7, 2010.

\begin{tabular}{r|l} 
ENDEREÇO & \\
Renato Barroso & \\
Escola de Educação Física e Esporte - USP & Recebido para publicação: 19/11/2009 \\
Av. Prof. Mello Moares, 65 & 1a. Revisão: 29/09/2010 \\
05508-030 - São Paulo - SP - BRASIL & 2a. Revisão: 20/10/2010 \\
e-mail: barroso@usp.br & Aceito: 21/ 10/2010 \\
&
\end{tabular}

